\title{
Report of a Rare Case of Sarcomatoid Renal Cell Carcinoma
}

\author{
Mohammad Ismail Hossain ${ }^{1 *}$ \\ Imtiaz Ahmed' \\ Pradip Bhattachariee \\ M Shahab Uddin Ahamad ${ }^{2}$ \\ Zillur Rahman \\ 'Department of Pathology \\ Chittagong Medical College \\ Chittagong, Bangladesh. \\ ${ }^{2}$ Department of Pathology \\ Cox's Bazar Medical College \\ Cox's Bazar, Bangladesh.
}

${ }^{*}$ Correspondence to:

\section{Mohammad Ismail Hossain}

Lecturer

Department of Pathology

Chittagong Medical College

Chittagong, 4203, Bangladesh.

Mobile: +88001712681719

E-mail: ismail.tushar@gmail.com

\begin{abstract}
A 50 years old non-smoker female with the history of right flank pain and hematuria surgically treated with right sided radical nephrectomy. Macroscopically it showed a large growth located at the lower pole of the right kidney. Histologically the tumor was composed of exuberant sarcomatous elements predominantly malignant fibrous histiocytoma like pattern and diagnosed as sarcomatoid renal cell carcinoma. There was no scope for immunohistological analysis of that tumor in our setup. It is important to recognize the sarcomatoid component in the renal tumors because of its aggressive behavior and adverse prognosis. We report herein a case of sarcomatoid renal cell carcinoma histomorphogically.
\end{abstract}

Key words: Kidney; Renal Cell Carcinoma; Sarcomatoid.

\section{INTRODUCTION}

The past two decades have seen dramatic changes in our understanding and management of renal cell carcinoma (RCC). Renal tumors with sarcoma-like appearances were characterized by pathologists as renal sarcomas in the early half of the 20th century ${ }^{1}$. The concept of sarcomatoid renal cell carcinoma (SRCC) was first described by Farrow et al. in 1968. They suggested that the sarcomatoid portion is derived from metaplastic transformation of carcinoma. Sarcomatoid RCCs have also been referred to as metaplastic carcinomas or spindle cell carcinomas ${ }^{2}$. SRCC is currently defined in the 2004 World Health Organization (WHO) classification of renal tumors as any histologic type of RCC containing foci of high grade malignant spindle cells ${ }^{3}$. It has been reported that sarcomatoid renal cell carcinomas constitute approximately $1.0 \%$ to $4.8 \%$ of the total number of renal parenchymal tumors ${ }^{4}$. Sarcomatoid carcinoma is a rare high-grade malignant neoplasm that shows morphologic and immunohistochemical evidence of both epithelial and mesenchymal differentiation ${ }^{5}$. Sarcomatoid carcinoma has been reported in a variety of organs, including the urinary bladder, kidney and female genital tract ${ }^{6}$. Renal cell carcinoma with a sarcomatoid component often shows local invasion and distant metastasis. The prognosis is worse than that of other types of tumors with a median survival time of 6.8 month?

Here, we histomorphologically present one such case in which the tumor showed RCC with extensive sarcomatous changes containing homologous malignant fibrous histiocytoma like differentiation.

\section{CASE REPORT}

A 50 years old non-smoker housewife presented with a 3 month long history of right flank pain and haematuria. There were no pertinent physical findings. Urine cytology revealed many RBCs and pus cells, but no malignant or atypical cells. Blood biochemistry showed moderate anemia. Computed tomography (CT) scan and USG done accordingly. CT findings showed a large heterogeneous mass at the lower pole of right kidney. Under the clinical diagnosis of renal cell carcinoma (RCC), she underwent right radical nephrectomy. 
Macroscopically the specimen consisted of right kidney surrounded by perinephric adipose tissue measuring $13.0 \mathrm{X}$ $10.0 \times 5.0 \mathrm{~cm}$. A $11.0 \times 9.0 \times 4.0 \mathrm{~cm}$ mass occupied most of the lower pole and displayed a gray-tan tumor with areas of necrosis (about $10 \%$ of the tumor). Apparently tumor not invaded the perinephric adipose tissues. Renal pelvis, ureter and renal vein were grossly free of tumor. The renal cortical tissue was compressed with uniform thinning. The adrenal gland was normal and free of tumor.

Microscopically, the tumor showed exuberant sarcomatoid differentiation: $90 \%$ of the tumor consisted of a malignant mesenchymal component with some areas of clear cell changes. The sarcomatoid cells are spindle, pleomorphic, hyperchromatic, with fair number of abnormal mitosis and multinucleated cells. The cells were arranged in ill-defined fascicles, with storiform pattern resembling malignant fibrous histiocytoma (MFH) like pattern (Figures 1-5). Sections from the renal vein, pelvis, ureter, adrenal gland and perinephric adipose tissue were free of tumor (Stage: T3 $\mathrm{Nx} \mathrm{Mx}$ ). Lymphovascular invasion was not seen. Fuhrman nuclear grade was G4. Immunohistochemical profile not possible to done because of unavailability of this technique in our setup.

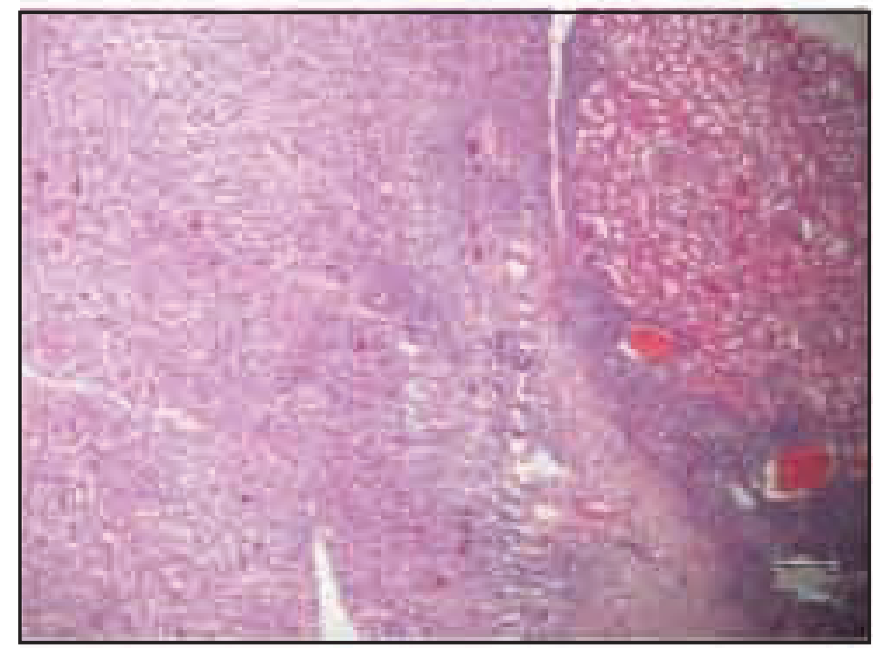

Figure 1: Tumor \& Kidney tissue interface

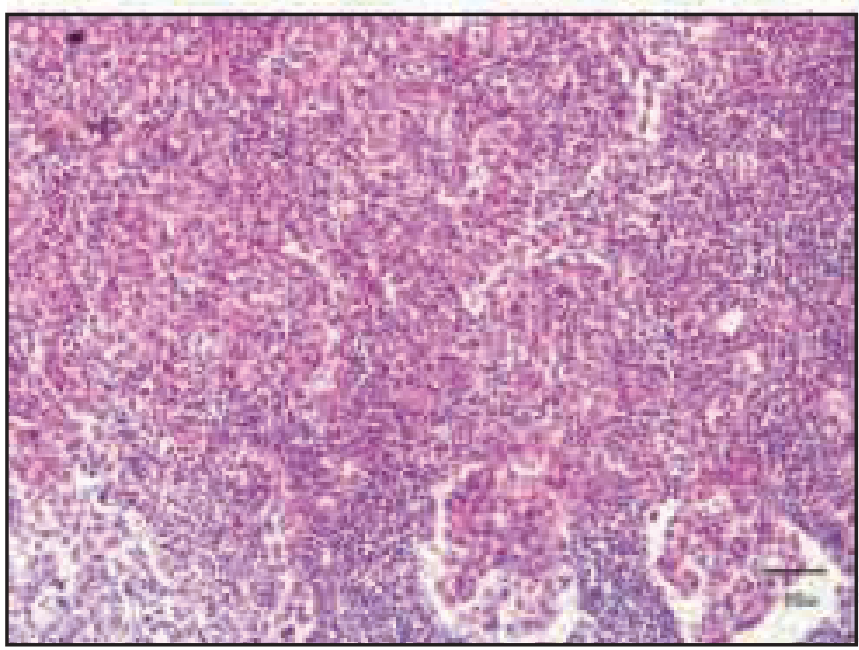

Figure 2 : Tumor along with two Glomeruli

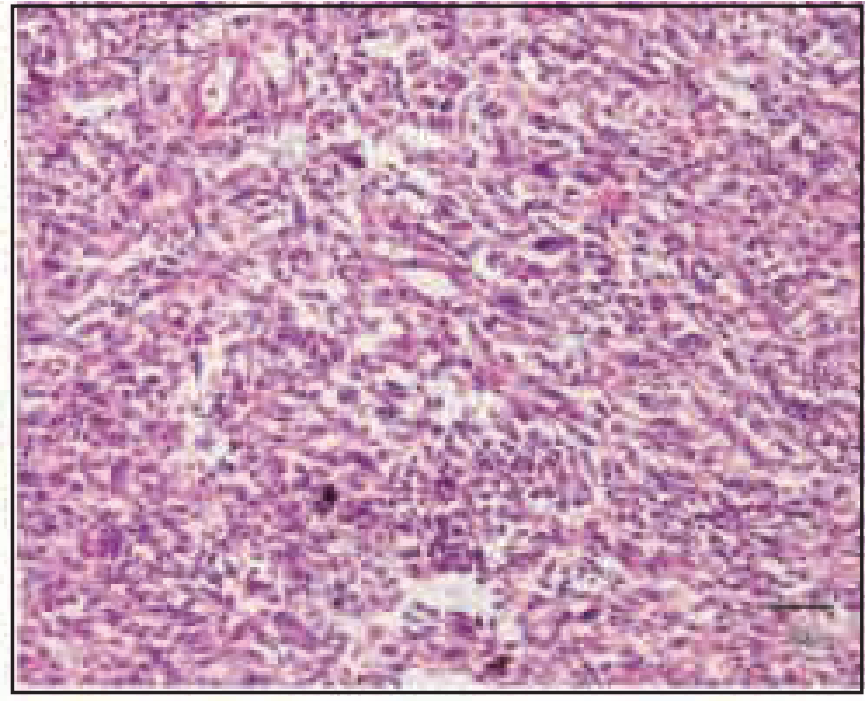

Figure 3 : Storiform growth low power view

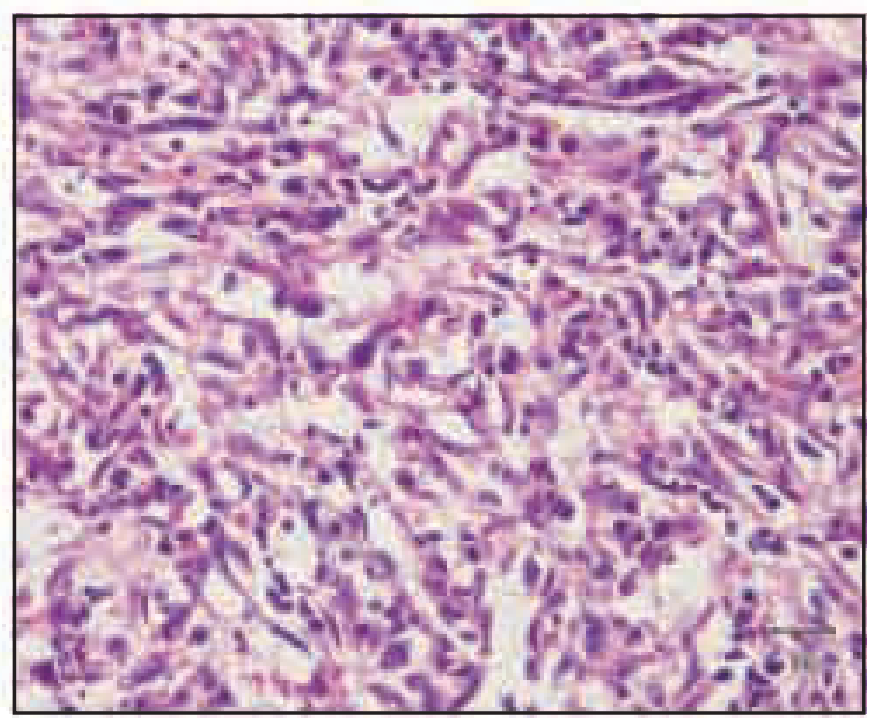

Figure 4 : Sarcomatous growth high power view

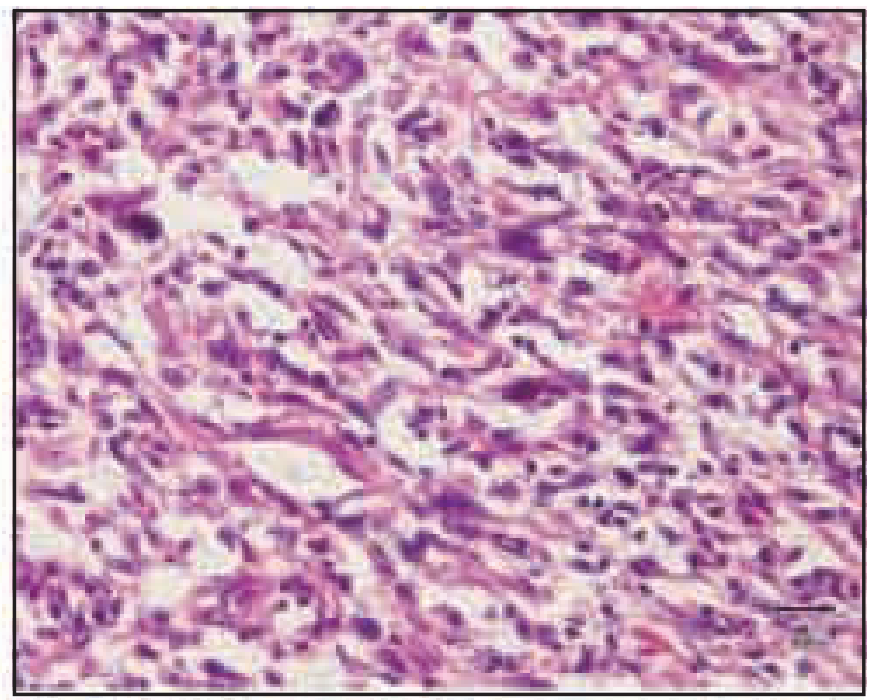

Figure 5: MFH like pattern with tumor giant cell

Volume 13, Issue 3, September 2014 


\section{DISCUSSION}

In this report we have described an exceedingly rare case of sarcomatoid renal cell carcinoma consisting mainly of malignant mesenchymal tumor with malignant fibrous histiocytoma like differentiation without any immunohistochemical profile. To our knowledge, fewer cases of sarcomatoid renal cell carcinomas have been reported. In most of these cases, the sarcomatous element consisted of poorly formed fascicles of undifferentiated spindle cells. However our case showed exuberant MFH like differentiation in most sarcomatous element.

Renal cell carcinoma is the sixth most common neoplasm in adults and accounts for $3 \%$ of adult malignancies and $90 \%$ of renal cancers. Sarcomatoid renal cell carcinoma initially felt to represent a primary renal sarcoma; it is now considered a form of dedifferentiated carcinoma and is therefore not a distinct histologic entity. Here is a summary table of sarcomatoid renal cell carcinoma (Table-1) ${ }^{8}$.

Table 1 : Summary table for sarcomatoid renal cell carcinoma

$\begin{array}{ll}\text { Etiology } & \text { Renal cell carcinoma occurs in both sporadic } \\ \text { (non-hereditary) and hereditary forms }\end{array}$

Incidence Sarcomatoid component reported to occur in $1-8 \%$ of renal cell carcinomas

Gender ratio Renal cell carcinoma is slightly more common in men, 1.6:1 male:female ratio

Age predilection No age predilection, renal cell carcinoma is most common in patients older than 50

Risk factors Obesity and cigarette smoking are strongest associated risk factors for renal cell carcinoma

Treatment Nephrectomy is primary therapy for renal cell carcinoma. With sarcomatoid component, use of chemotherapy agents has shown success in prolonging patient survival

Prognosis Very poor prognosis for sarcomatoid renal cell carcinoma with median survival less than 1 year

Imaging features Sarcomatoid renal cell carcinoma typically demonstrates aggressive features, such as necrosis, renal vein invasion and renal sinus invasion

This process is thought to result from dedifferentiation of the epithelial component, and so the sarcomatoid cells are expected to show the original genomic pattern of the parent cells.
$\mathrm{X}$-chromosome inactivation analysis has confirmed this hypothesis by showing different patterns of allelic loss in multiple chromosomal regions, indicating divergence during the clonal evolution of $\mathrm{RCC}^{9}$.

Sarcomatoid RCCs arise from all types of RCCs including conventional, papillary, chromophobe, and collecting duct carcinomas. With regard to the growth pattern of the sarcomatoid component, malignant fibrous histiocytomatous, fibrosarcomatous, unclassified sarcomatous, and hemangiopericytomatous patterns have been identified. Immunohistochemically, more than half of sarcomatoid RCCs are positive for AE1/AE3, epithelial membrane antigen (EMA) and vimentin. However, sarcomatoid variants show stronger staining in a higher proportion of cases for vimentin and reduced positivity for EMA compared with conventional RCCs. Staining for other mesenchymal markers such as desmin, actin and S-100 is generally negative ${ }^{2}$.

Sarcomatoid carcinoma is a high grade neoplasm. The exact pathogenesis of sarcomatoid carcinoma is not known. Two opposing theories have been proposed. The monoclonal theory states that the carcinomatous and sarcomatous tumor cells are both derived from a single pluripotent stem cell that undergoes divergent epithelial and mesenchymal differentiation. The multiclonal theory states that the sarcomatoid carcinoma is a collision tumor composed of the derivatives of two or more stem cells of separate epithelial and mesenchymal origin. The main differential diagnosis includes true sarcoma and pseudosarcomatous mesenchymal proliferations. Mixed malignant epithelial and mesenchymal components are diagnostic, and immunohistochemical staining for epithelial and mesenchymal markers is helpful ${ }^{6}$.

Many studies have defined a tumor as sarcomatoid renal cell carcinoma (SRCC) if even a small amount of sarcomatoid differentiation is present However, some evidence exists of increased risk associated with sarcomatoid components comprising $5-10 \%$ of total tumor volume, indicating that even small amounts of sarcomatoid differentiation may be clinically relevant and should be included in the pathology report ${ }^{3}$.

In conclusion, we described a rare case of sarcomatoid renal cell carcinoma with predominantly malignant fibrous histiocytoma like pattern histomorphologically. Although this tumor is very rare, sarcomatoid carcinoma should be included in the differential diagnosis of the renal tumor.

\section{DISCLOSURE}

All the authors declared no competing interest. 


\section{REFERENCES}

1. Shuch B, Bratslavsky G, Linehan WM, Srinivasan R. Sarcomatoid Renal Cell Carcinoma: a Comprehensive Review of the Biology and Current Treatment Strategies. The Oncologist. 2012; 17: 46-54.

2. Kuroda N, Toi M, Hiroi M, Enzan H. Review of sarcomatoid renal cell carcinoma with focus on clinical and pathobiological aspects. Histol Histopathol. 2003; 18: 551-555.

3. Roohi M, Tanvir I, Qazi S. Sarcomatoid Renal Cell Carcinoma. International J Pathol. 2012; 10(1): 39-40.

4. UZUNLAR AK, OZDEMIR E, YALDIZ M, Kaya O. Sarcomatoid Renal Cell Carcinoma and its Chromophobe Connection. Turk J Med Sci. 2000; 30: 505-507.

5. Chen S, Chen G, Xia D, Li J, Wang S, Shen B, Jin B. Sarcomatoid carcinoma of the renal pelvis: Experience of multiple cases over a ten year period. ONCOLOGY LETTERS. 2013; 6: 513-516. DOI: 10.3892/ol.2013.1396

6. Ahn HI, Sim J, Han H, Kim H, Yi K, Jun YJ, Rehman A, Jang SM, Jang K, Paik SS. A Case of a Sarcomatoid Carcinoma Arising in the Renal Pelvis with Exuberant Osteosarcomatous Element. Open J Pathol. 2013; 3: 96-98.

7. Murakami K, Yamamoto K, Aoki K, Fukumoto I, Sugiura T, Kirita T. Metastatic sarcomatoid renal cell carcinoma to the mandible treated with Sorafenib. Open J Stomatol. 2012; 2: 22-27.

8. Reiter M, Schwope R, Clarkson A. Sarcomatoid renal cell carcinoma: A case report and literature review. Radiology Case. 2012; 6(4): 11-16.

9. Quiroga-Garza G, Khurana H, Shen S, Ayala AG, Ro JY. Sarcomatoid chromophobe renal cell carcinoma with heterologus sarcomatoid elements. Arch Pathol Lab Med. 2009; 133: 1857-1860. 